\title{
DISEÑO DE ENTORNOS DE APRENDIZAJE COLABORATIVOS, MEDIANTE EL USO DE LAS TAC Y TEP
}

\author{
Carrera Pola Maritza ${ }^{1}$, Toledo Santos Beatriz ${ }^{2}$, Ramírez Carrera Elisa Carolina ${ }^{3}$
}

\section{RESUMEN}

El presente artículo presenta la experiencia educativa aplicando las tecnologías de aprendizaje y conocimiento (TAC), mediante el aprendizaje basado en proyectos, para llegar a las tecnologías del empoderamiento y participación (TEP). Esta propuesta consistió en la organización y creación de dos sitios web 2.0 y el uso de rede sociales para la educación en salud, de problemas de salud que aquejan a la sociedad en México y principalmente en Chiapas. Los participantes fueron 30 alumnos del módulo II de la licenciatura de Médico Cirujano, de la Facultad de Medicina Humana campus II. Se enmarca en la metodología de investigación-acción. El desarrollo del proyecto fue coordinado por los docentes participantes y fue conducido autónomamente por los propios estudiantes, durante un semestre. Los resultados obtenidos muestran el alcance del proyecto como un poderoso recurso para alcanzar los objetivos en competencias curriculares del programa de estudios que sitúan a los estudiantes en un entorno escolar en la que puedan ser críticos, reflexivos y que les concede espacios de autonomía para el desarrollo académico y social, a través de la participación en la sociedad, logrando el empoderamiento como estudiantes buscando mejorar la calidad de vida y salud de las personas mediante la educación utilizando las recursos de las redes sociales y entornos web 2.0.

PALABRAS CLAVE: Aprendizaje basado en proyectos, Promoción de la Salud, TAC, TEP.

\section{ABSTRACT}

This article presents the educational experience by applying learning and knowledge technologies (TAC), through project-based learning, to reach the technologies of empowerment and participation (TEP). This proposal consisted in the organization and creation of two 2.0 websites and the use of social networks for

\footnotetext{
${ }^{1}$ Universidad Autónoma de Chiapas.

${ }^{2}$ Universidad Autónoma de Chiapas.

${ }^{3}$ Instituto Dermatológico de Jalisco.
}

\begin{tabular}{c|c|c|c}
\hline Rev. Ciências Humanas & Frederico Westphalen, RS & Pg. $57-68$ & Set./dez. 2018 \\
\hline \multicolumn{2}{c|}{ Recebido em: 04/01/2018 } & Aceito em: 04/02/2019 \\
\hline
\end{tabular}




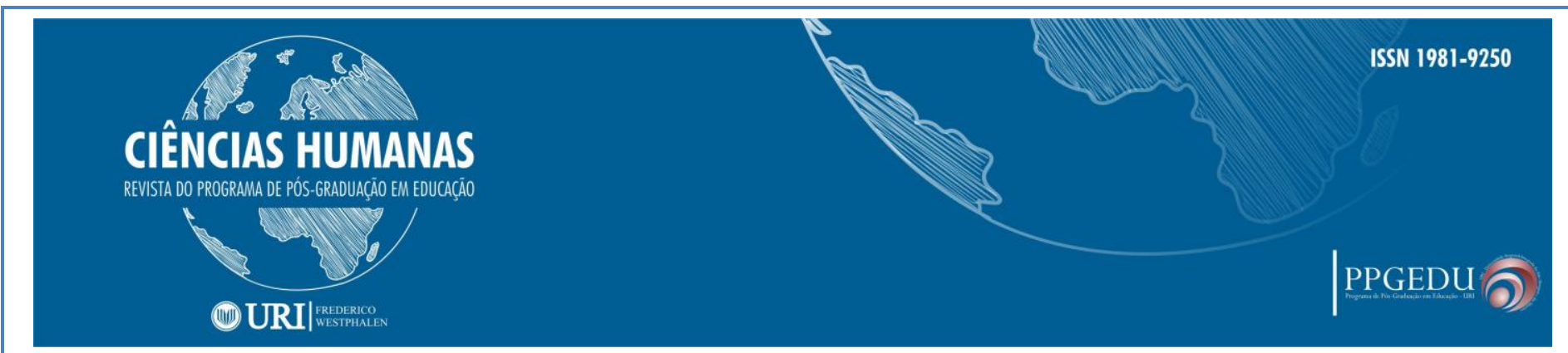

health education, health problems that afflict society in Mexico and mainly in Chiapas. The participants were 30 students of module II of the degree of Medical Surgeon, of the Faculty of Human Medicine campus II. It is part of the action research methodology. The development of the project was coordinated by the participating teachers and was conducted autonomously by the students themselves, during a semester. The obtained results show the scope of the project as a powerful resource to reach the objectives in curricular competitions of the study program that place the students in a school environment in which they can be critical, reflective and that grants them spaces of autonomy for the development academic and social, through participation in society, achieving empowerment as students seeking to improve the quality of life and health of people through education using the resources of social networks and web 2.0 environments.

KEYWORDS: Project-based learning, Health Promotion, TAC, TEP.

\section{INTRODUCCIÓN}

La salud es un derecho inalienable del ser humano, una población sana para un país significa la posibilidad de ser una población activa, capaz de generar su propio bienestar y tomar sus propias decisiones, activa en el contexto económico y democrático de un país. Es una garantía constitucional que se acuña en el artículo $4^{\circ}$ de la Carta Magna.

El otro pilar de gran importancia es la educación, esta da la posibilidad a las personas de abrir sus expectativas, buscando la libertad, a través del acceso al conocimiento. La unión de ambos conceptos: salud y educación, posibilitan que las personas puedan adquirir el empoderamiento de estos derechos (Binde, 2005, P. 155).

La Carta de Ottawa de 1986, OMS. (1986), dice: “La promoción de la salud consiste en proporcionar a los pueblos los medios necesarios para mejorar su salud y ejercer un mayor control sobre la misma. Para alcanzar un estado adecuado de bienestar físico, mental y social un individuo o grupo debe ser capaz de identificar y realizar sus aspiraciones, de satisfacer sus necesidades y de cambiar o adaptarse al medio ambiente. La salud se percibe pues, no como el objetivo, sino como la fuente de riqueza de la vida cotidiana. Se trata por tanto de un concepto positivo que acentúa los recursos sociales y personales así como las aptitudes físicas. Por consiguiente, dado

\begin{tabular}{c|c|c|c}
\hline Rev. Ciências Humanas & Frederico Westphalen, RS & Pg. 57 - 68 & Set./dez. 2018 \\
\hline \multicolumn{2}{c}{ Recebido em: 04/01/2018 } & Aceito em: 04/02/2019 \\
\hline
\end{tabular}




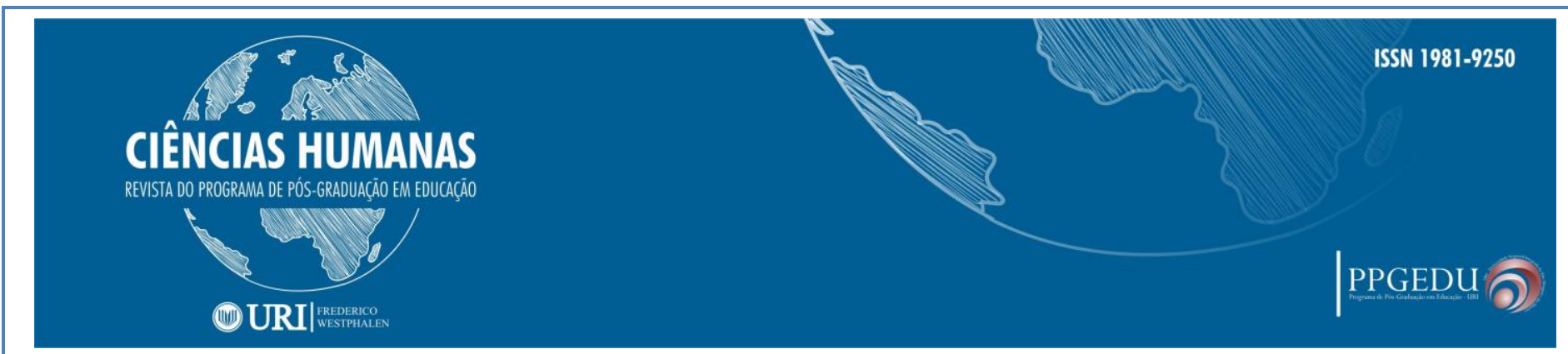

que el concepto de salud como bienestar trasciende la idea de formas de vida sanas, la promoción de la salud no concierne exclusivamente al sector sanitario”. (p.1).

Esta propuesta audaz y diferente propuso utilizar internet, radio, redes sociales y móviles, como una visión integral y diferente del modelo de sanidad. Básicamente consiste en la creación de nuevos sistemas de información diseñados para interactuar de forma ágil, ubicua, sencilla, y pensados para ser usados por los profesionales médicos, estudiantes de medicina y los ciudadanos. Se sustenta en el concepto de tecnologías de aprendizaje y conocimiento (TAC), a través de la utilización de web 2.0 que incluye numerosas herramientas gratuitas con un amplio abanico de posibilidades de comunicación que pueden serán utilizadas en la relación entre profesionales sanitarios o como aplicación directa a los pacientes, permitiendo el uso de estas tecnologías para el empoderamiento en salud de las personas.

\section{DESARROLLO}

\section{OBJETIVO GENERAL.}

Utilizar las tecnologías de aprendizaje y conocimiento (TAC), mediante el aprendizaje basado en proyectos, para que el estudiante de medicina incida en la problemática en salud logrando su participación social, y su empoderamiento al desarrollar sus capacidades y potencialidades buscando la relación de éstas con el entorno que le rodea.

\section{MATERIALES Y MÉTODOS}

Se realizó una investigación pedagógica, en el ciclo escolar enero junio 2016, en la Facultad de Medicina Humana C-II, en el módulo II "El hombre sano y su entorno II", para lo cual se diseñaron y realizaron proyectos para realizar páginas web interactivas con temáticas acordes a la promoción de la salud, la cual junto con las competencias en Tecnologías de la Información y Comunicación forman parte del programa de estudios y del perfil de egreso de los estudiantes de Medicina. (Gómez, J. 2013), la visión de la Facultad señala: “...formación de profesionales de la salud con excelente calidad,

\begin{tabular}{c|c|c|c}
\hline Rev. Ciências Humanas & Frederico Westphalen, RS & Pg. 57 - 68 & Set./dez. 2018 \\
\hline Recebido em: 04/01/2018 & Aceito em: 04/02/2019 \\
\hline
\end{tabular}




\section{CIÊNCIAS HUMANAS}

REVISTA DO PROGRAMA DE PÓS-GRADUACĀO EM EDUCAÇĀO

\section{(1) URI|}

mediante educación innovadora e investigación creativa y recreativa, aplicadas y con alto impacto en el bienestar de la población del estado de Chiapas y del país" (p.3), lo cual se cumple satisfactoriamente a través de estos trabajos.

El universo de trabajo estuvo constituido por 30 estudiantes del Módulo II de la Licenciatura de Médico Cirujano del ciclo escolar enero junio 2016.

Debido a que durante el desarrollo de las actividades de investigación y docencia, se presentaban dos enfermedades emergentes en el país y principalmente en Chiapas, se abordaron los siguientes problemas de salud:

1. Chikungunya.

2. Zika.

Para el desarrollo de ambos proyectos se recurrió al el aprendizaje basado en proyectos, y se establecieron las siguientes acciones:

1. Desarrollo de habilidades informacionales: mediante una exhaustiva revisión en bases de datos especializadas sobre las enfermedades de Chikungunya y Zika.

2. Conformación de los equipos colaborativos de trabajo, los cuales estarían subdivididos en áreas especiales. Un coordinador general, un equipo de Multimedia (video, audio), equipo de diseño de página web, equipo de redes sociales, equipo de investigación.

3. Se identificó a los subcoordinadores por cada área específica.

4. Se estableció el plan de trabajo, cronograma de actividades.

5. Se establecieron los tiempos de entrega y cada equipo iniciaron los trabajos, se buscó la colaboración de tutores maestros médicos para que dieran asesoría sobre el tema a los alumnos.

6. Se estableció la fecha de presentación de los proyectos.

Las vivencias obtenidas y las opiniones expresadas por alumnos y profesores fueron la base de los resultados de estos proyectos realizados; se expresaron los valores en 


\section{CIÊNCIAS HUMANAS}

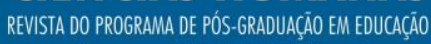

\section{(1) URI|}

frecuencias absolutas y porcentajes, y se aplicaron métodos propios de la estadística descriptiva.

\section{RESULTADOS}

Como investigadores consideramos de suma importancia trasladar y crear un ambiente propicio para la construcción del conocimiento, con aprendizajes significativos de los estudiante.

Los jóvenes a su vez buscaron la comunicación y difusión de la información creada a través de innovadoras imágenes, vídeos y podcast, mediante páginas web y redes sociales a toda la población interesada.

Para ello fue necesario contar con la asesoría de una especialista médica en Epidemiología, también se realizó una investigación bibliográfica a través de bases de datos especializadas (Ebsco Host, CONRICYT) y sitios web gubernamentales: Sitio Oficial de la Secretaria de Salud México, y organismos internaciones como la OMS y OPS.

Se construyeron los sitios web utilizando la utilería en línea WIX, por ser una plataforma sencilla, con plantillas diseñadas por una comunidad de expertos en el diseño de páginas web, además de su bajo costo.

Para el proyecto de Zika se creo el siguiente sitio: http://inforzikamed.wixsite.com/informedicos

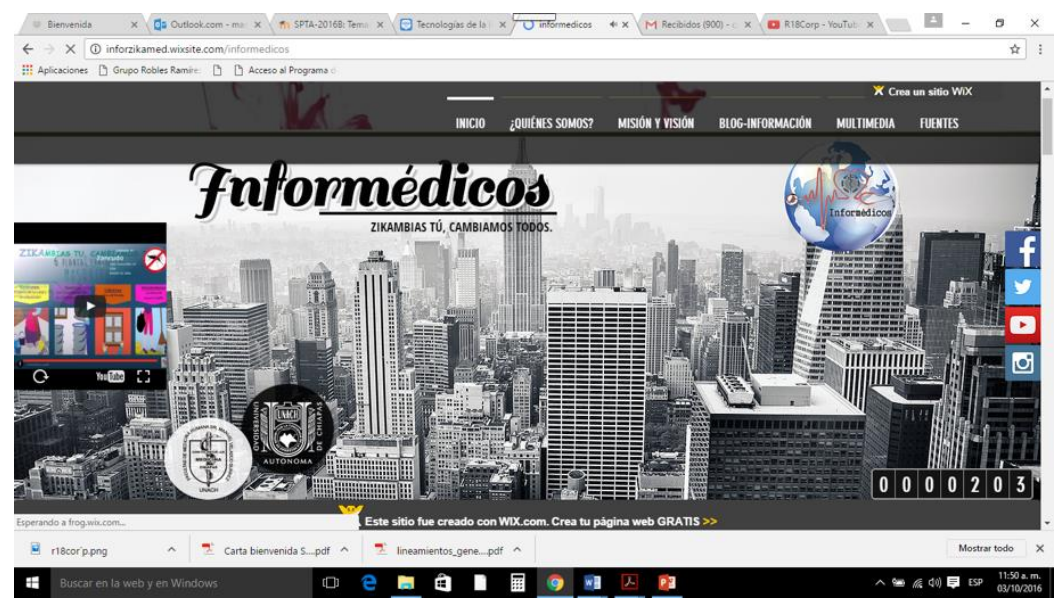

Figura 1. Sitio web de Informédicos 


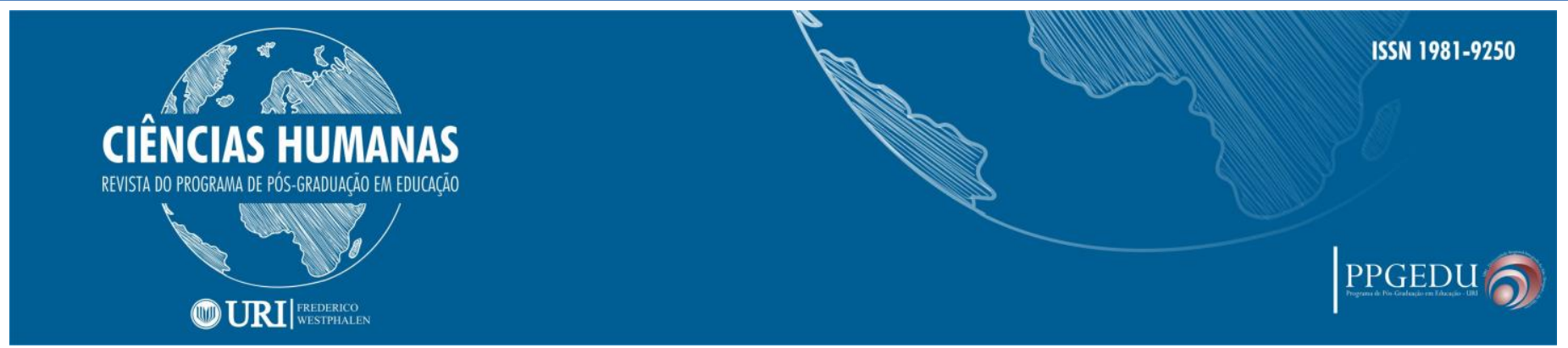

Para el proyecto de Chikungunya, el equipo denominado Innova Med creo su sitio: http://innovamed.mex.tl/frameset.php?url=/, ver Figura 2.

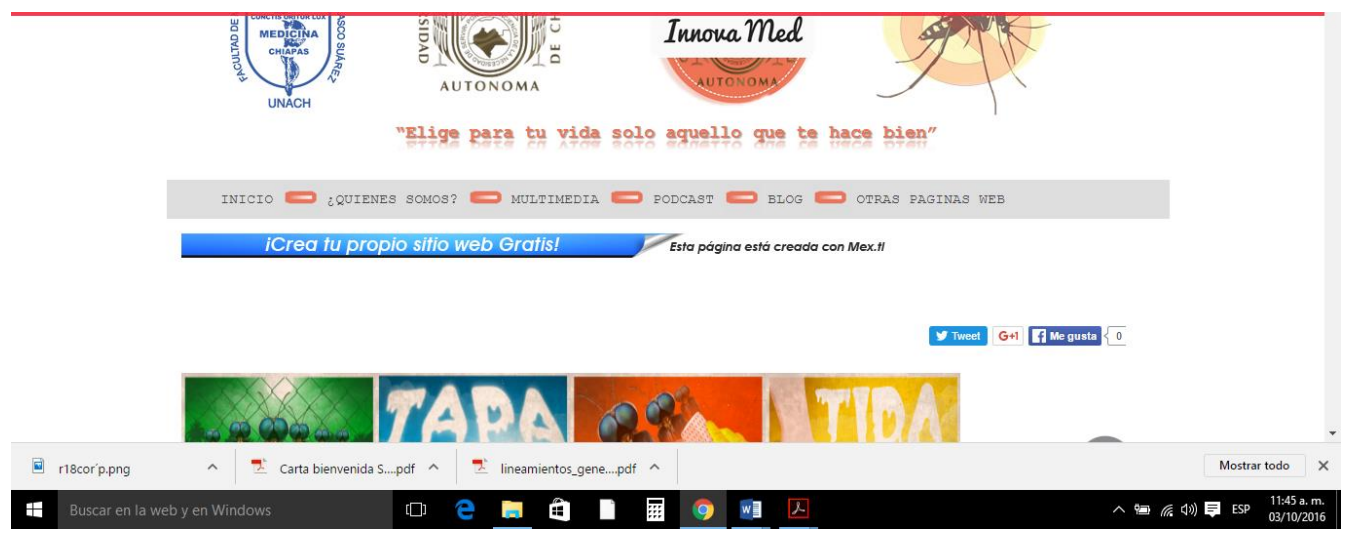

Figura 2. Sitio web de Innova Med.

Para un fácil acceso a la información se crearon las siguientes opciones del menú:

Inicio: La página principal con el logo y lema que dan identidad al equipo.
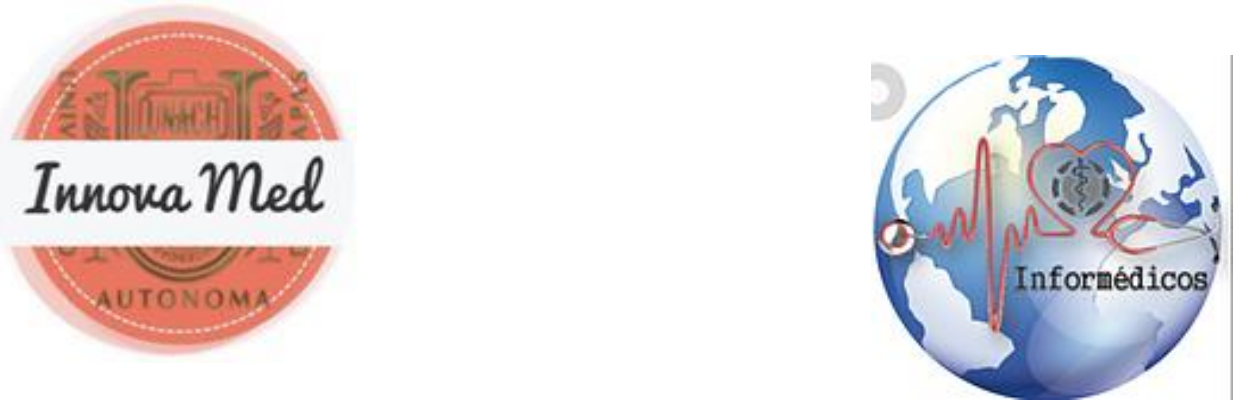

Figura. 3 Logo del equipo Innova Med

Figura 4. Logo del equipo de Informédicos 


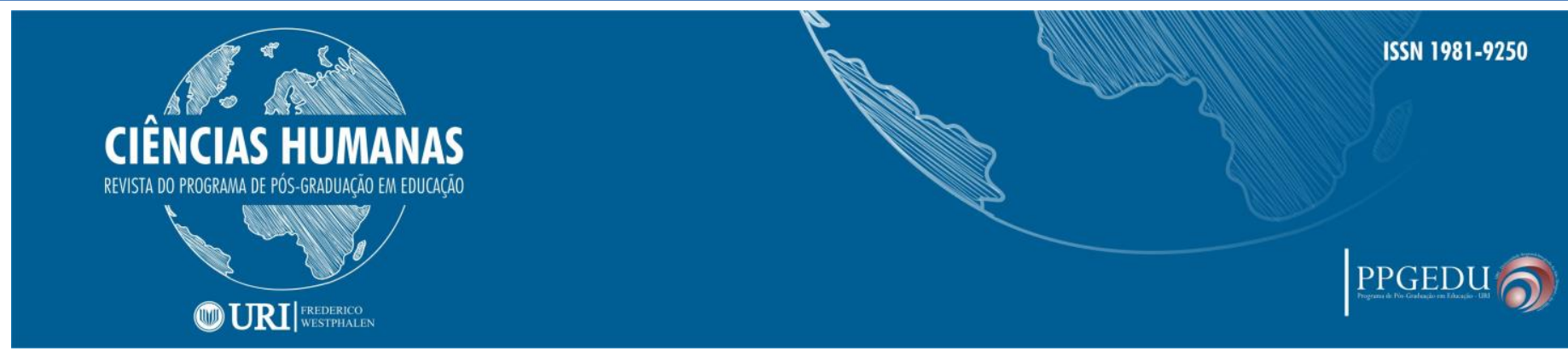

También se colocó la bienvenida e información en formato escrito y de forma oral a través de un podcast.

Quiénes somos: El equipo se presentó como un grupo colaborativo que crea el proyecto, estableciendo la información correspondiente.

Blog informativo: En el blog informativo se colocaron los resultados de la investigación bibliográfica, así como hipervínculos a sitios de información gubernamental y de otras organizaciones internacionales dedicadas a la salud.

Así como literatura que pudiera ahondar en el tema desde bases de datos especializadas.

\section{Multimedia:}

Videos. Se creó un canal de YouTube para compartir los videos, creados a exprofeso por los alumnos, utilizando como software: Sony Vega y iMovie.

Podcast. Se creó un canal de Podcast en iVox, aquí se colocándose las cápsulas informativas, las cuales son grabadas y editadas utilizando el software de Audacity.

\section{Redes sociales:}

Se crearon cuentas en Facebook, Twitter e Instagram, por cada uno de los equipos involucrados.
A) Facebook 

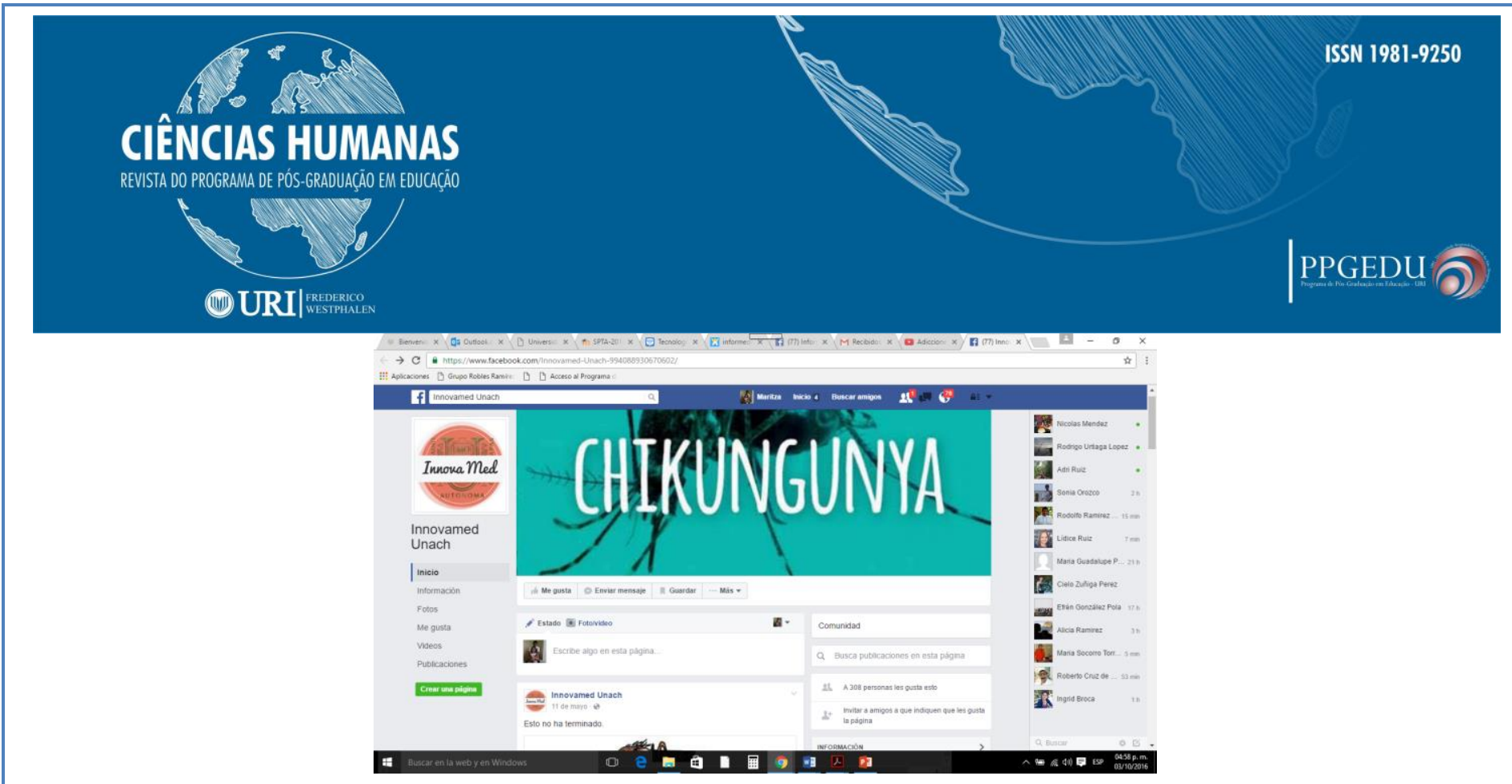

Figura 5. Red social Facebook para el proyecto de Chikungunya

En el proyecto "Zika una enfermedad emergente prevención y cuidados", se tuvieron las siguientes actividades de promoción en línea:

$\checkmark$ La página de Facebook fue nombrada como "Informédicos", con un total de 588 seguidores hasta el 15 de marzo del 2016. Con un alcance de 2,000 personas, en su mayoría mujeres de 18 a 24 años.

$\checkmark$ La dirección electrónica es: https://www.facebook.com/informedicos.unach/

Mientras que para el Proyecto "Chikungunya, una enfermedad emergente, prevención y cuidados", la página de Facebook se nombró: "Innovamed”, con 308 seguidores, ver figura 5.

\section{B) Instagram}

En la cuenta de esta red social, se dedicaron a postear imágenes con fines informativos, así como aquellas fotografías que dieran ímpetu y carácter a los equipos. Se alcanzó la cifra de 38 seguidores para el caso del proyecto de Zika, y en el proyecto de Chikungunya 92 seguidores. 


\section{C) Twitter}

En esta red social se agregó contenido de tipo informativo durante las fechas los meses de marzo a mayo, llegando a los 28 seguidores. https://twitter.com/Informedicos_ZK, y en el caso del proyecto de Zika a través de https://twitter.com/innovamed2c, obtuvieron 10 seguidores.

\section{D) YouTube}

Los vídeos en el canal de YouTube para el proyecto de Zika, alcanzaron 68 visitas, varias suscripciones. Canal de YouTube: https://www.youtube.com/watch?v=46MLL0Gf6gU

En el caso del proyecto de Chikungunya, en el canal de YouTube, se alcanzaron las cifras de 2 suscriptores y 54 vistas, Canal de YouTube: https://www.youtube.com/channel/UCJ7EotsfF8s2zsMPVyrjMEQ

\section{E) IVOX}

Mientras que en el canal de iVox se subieron 5 cápsulas informativas y se puede seguir en la dirección: http://www.ivoox.com/r18corp_aj_4965489_1.html

\section{CONCLUSIONES}

1. La educación en el aula debe estar en una constante actividad, deben romper paradigmas, buscando nuevos esquemas para el proceso de enseñanza aprendizaje, de manera tal que se busque el accionar de los estudiantes, para que ellos se vuelvan propositivos, creativos e innovadores, construyendo su propio conocimiento a través de la investigación, promoviendo el trabajo grupal y

\begin{tabular}{c|c|c|c}
\hline Rev. Ciências Humanas & Frederico Westphalen, RS & Pg. 57 - 68 & Set./dez. 2018 \\
\hline \multicolumn{2}{c}{ Recebido em: 04/01/2018 } & \multicolumn{3}{c}{ Aceito em: 04/02/2019 } \\
\hline
\end{tabular}




\section{CIÊNCIAS HUMANAS}

\section{(1) URI|}

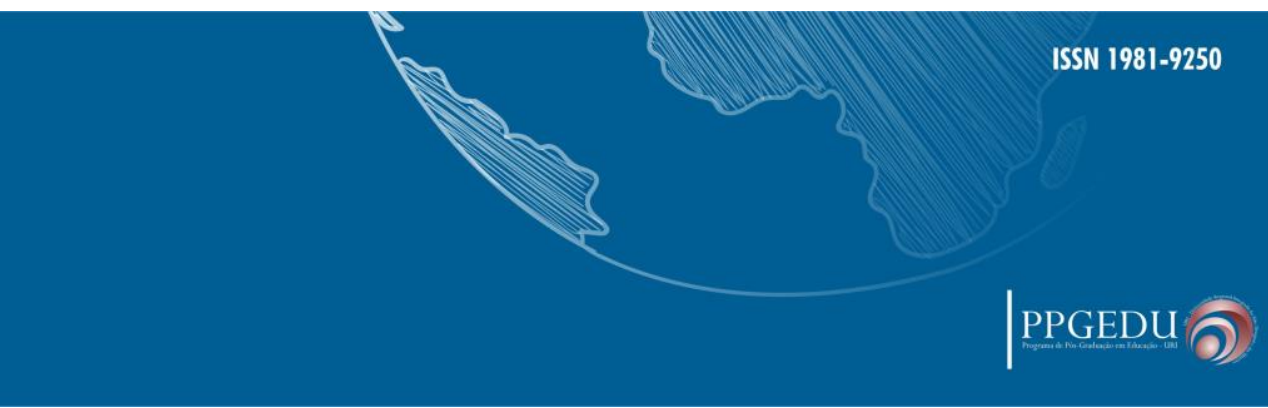

colaborativo, buscando el aprender a aprender, aprender a hacer, el saber ser, y aprender a convivir.

2. El aprendizaje basado en proyectos, permite al estudiante el hacer como una forma más fácil de aprender. (Area, 2008)

3. El joven estudiante de este siglo, es un nativo digital, cuya comprensión del manejo y apropiación de las tecnologías de la información y las comunicaciones (TIC) son competencias para su desarrollo como individuos dentro de las sociedades, que les permiten el manejo de la información, para crear, compartir, modificar, enriquecer la información en conocimiento. (Matarín, 2014)

4. El reto entonces para el maestro es motivar a los estudiante para que utilicen estas herramientas de forma didáctica en la construcción del conocimiento, es decir transformar en el aula las TIC en TAC, mediante su uso en para la construcción del conocimiento.

5. Para ello es necesario incluir el uso de las redes sociales, motivando a los jóvenes a buscar la colaboración y participación con su entorno social, haciéndole incapie que la educación intramuros debe ser extramuros, y que es parte del conociento el incluir la problemática social en su localidad, nación y del mundo.

6. A través de esta experiencia, instrumentando el uso y aprovechamiento de las tecnologías del aprendizaje y conocimiento (TAC), mediante la creación de espacios en la WEB 2.0 y las redes sociales más conocidas como Facebook, Instagram, Twitter, YouTube, iVox, se buscó que los jóvenes entendieran y aprovecharán de forma innovadora e intelige la promoción de la salud, para apoyar a la sociedad para que mediante la educación se hiciera una campaña de prevención para atacar en este caso la propagación de las enfermedades de Chikungunya y Zika, que por las situaciones geográficas de Chiapas, como entidad fronteriza de México estaba padeciendo, y los estudiante pudieran 


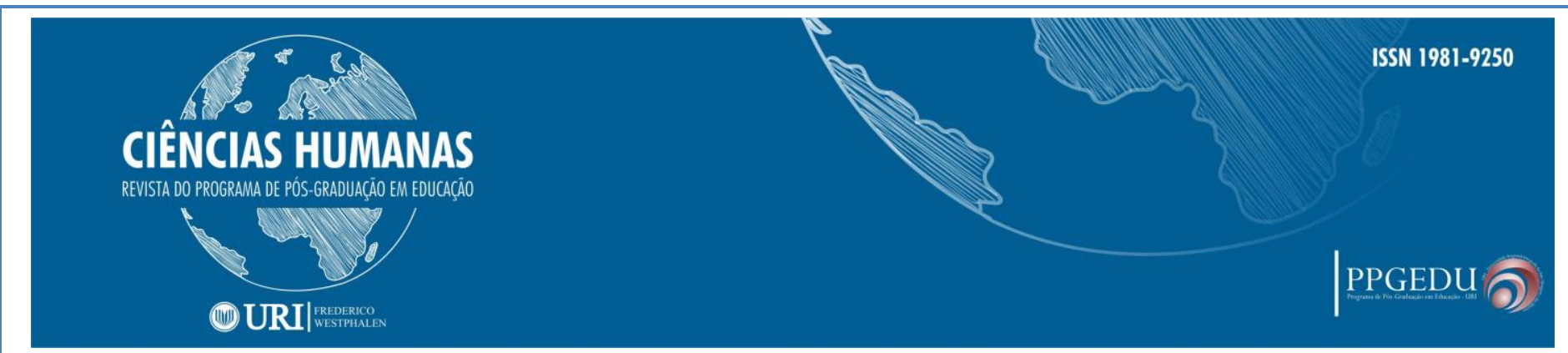

mediante sus fortalezas en el conocimento del uso tecnologías de la información y la comunicación, empoderarse para la creación de estos proyectos en donde se les dio una guía, tutoría y asesoramiento, pero que se les permitió crear e innovar y tomar decisiones.

7. Fue realmente inusitada la inspiración lograda, ya que al construir una identidad propia como equipos colaborativos de trabajo con la creación de un logo y un lema, los jóvenes se identificaron entre si buscando realizar un trabajo de calidad en pro del colectivo.

8. Las habilidades y destrezas mostradas en la realización de los proyectos muestran la capacidad que tienen los estudiantes para afrontar los retos, ya que estas mismas se requieren para el trabajo en la comunidad a través de la promoción de la salud, de la cual retomaron la importancia de acuerdo al momento de auge de las enfermedades emergentes Zika y Chikungunya. Cabe mencionar que en el proyecto de Chikungunya se logró además hacer una difusión más amplia a través de una radiodifusora en internet, que da cobertura a la zona de Comitán llegando hasta el país vecino de Guatemala.

9. Esta ha sido una experiencia muy enriquecedora, que nos lleva a buscar nuevos entornos y formas para lograr una educación de calidad, para formar a las nuevas generaciones del presente siglo.

\section{REFERENCIAS BIBLIOGRÁFICAS}

Area, M. (2008). La innovación pedagógica con TIC y el desarrollo de las competencias informacionales y digitales. Investigación en la Escuela, 5-17.

Bindé, J. (2005). Hacia las sociedades del conocimiento. París, Francia: Ediciones UNESCO.

\begin{tabular}{c|c|c|c}
\hline Rev. Ciências Humanas & Frederico Westphalen, RS & Pg. 57 - 68 & Set./dez. 2018 \\
\hline \multicolumn{2}{c}{ Recebido em: 04/01/2018 } & Aceito em: 04/02/2019 \\
\hline
\end{tabular}




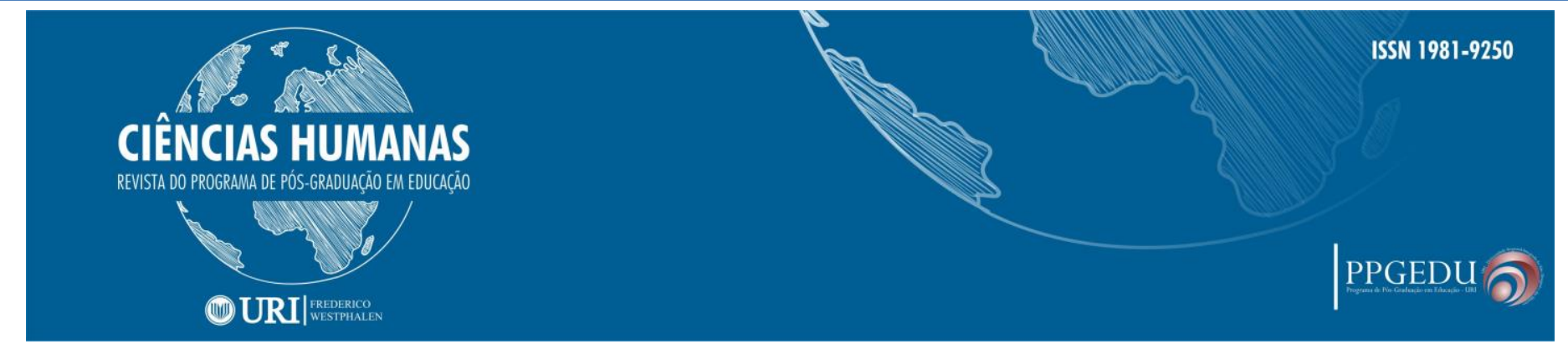

Matarín, T. (2014). Redes sociales en prevención y promoción de la salud. Una revisión de la actualidad. Revista española de comunicación en salud, 62-69.

OMS. (1986). Primera Conferencia Internacional sobre la Promoción de la Salud. agosto 9, 2018, de OPS Sitio web:

http://www1.paho.org/spanish/HPP/OttawaCharterSp.pdf

Gómez, J.. (2013). Plan de Estudios de la Licenciatura de Médico Cirujano 2013.

Septiembre 2, 2018, de UNACH Sitio web:

http://www.facmed.unach.mx/images/oferta/Lic/plan_med_2013.pdf 